\title{
H-DenseUNet for Kidney and Tumor Segmentation from CT Scans
}

\author{
Xiaomeng Li, Lihao Liu, Pheng-Ann Heng \\ Department of Computer Science and Engineering, \\ The Chinese University of Hong Kong, Hong Kong
}

\begin{abstract}
Automatic kidney tumor segmentation from CT scans is an essential step for computer-aided diagnosis of cancer. In this paper, we present an improved H-DenseUNet for kidney and tumor segmentation. Specifically, we first train the DenseUNet and then fine tune the network with the $3 \mathrm{D}$ counterpart. To further increase the performance, we employ both cross-entropy and dice loss. We evaluate our method on the 2019 MICCAI kidney and tumor segmentation challenge. We split the training dataset of the challenge to 200 training set and 10 validation set. On the validation set, our method achieves $97.0 \%$ (Dice) for kidney segmentation and $67.2 \%$ (Dice) for tumor segmentation. This model is submitted to the challenge for final performance evaluation on the test dataset.
\end{abstract}

\section{Introduction}

Kidney cancer is among the 10 most common cancers in both men and women. In 2019, an estimated 73,820 adults (44,120 men and 29,700 women) in the United States will be diagnosed with kidney cancer [5]. According to the clinical observation, the locations of different kidney tumors in medical images, i.e., CT or MR, are very difficult to predict, since kidney tumor has various size, shape, location and numbers within one patient. Furthermore, some lesions do not have clear boundaries and are extremely small. Due to the wide variety in kidney and kidney tumor morphology, there is currently great interest in how tumor morphology relates to surgical outcomes. Automatic semantic segmentation could help in this process, and thus is highly demanded in the clinical practice.

Recently, MICCAI 2019 kidney cancer segmentation challenge [1,3] is proposed to accelerate the development of reliable kidney and kidney tumor semantic segmentation methodologies. In this dataset, 300 unique kidney cancer CT scans are collected. 210 of these have been released for model training and validation, and the remaining 90 will be held out for objective model evaluation. The ground truth semantic segmentations masks for the training dataset are provided.

To develop an automatic kidney and cancer segmentation method, we participate this challenge. In this following, we describe our method in this challenge. 


\section{Preprocessing}

In the preprocessing, we truncated the image intensity values of all scans to the range of [-200, 250] HU to remove the irrelevant details. We did not employ any other preprocessing strategies.

\section{Architecture}

We employed H-DenseUNet [4] in this challenge, which consists of a 2D DenseUNet and a 3D counterpart. In this first stage, we train a 2D DenseUNet [4] for kidney and tumor segmentation simultaneously. The modification to the original framework is that we replace the SGD optimizer to Adam [2], with initial learning rate 0.001 . The learning rate is decayed every 100 epochs. In this second stage, we fine tune the tumor segmentation result with the 3D DenseUNet. The hybrid network is initialized with the model from the first stage. The optimizer is changed to Adam [2] and learning rate is 0.0003. The loss function is the weighted cross-entropy loss, and the class weight keeps the same with [4]. When model converges, we test our segmentation result for kidney and tumor, respectively.

\section{Inference and Post-processing}

The whole framework is implemented with Keras. For data augmentation, we adopted random mirror, random rotation with 90 degree and random scaling between 0.8 and 1.2 for all training data to alleviate the overfitting problem.

To train the 2D DenseUNet, the input images are sliced into three adjacent slices, and the input size is randomly cropped patches $(224 \times 224)$. The training of 2D DenseUNet model used 4 NVIDIA Titan Xp GPUs with 12 GB memory, with batch size 32 . We first train with cross-entropy loss, and then fine tune with the averaged of the cross-entropy loss and dice loss. The kidney segmentation result is generated by the $2 \mathrm{D}$ DenseUNet and tumor segmentation result is generated by the H-DenseUNet.

To test 2D DenseUNet, we test the volume in a slice-by-slice manner. We first resize the input three adjacent slices to $512 \times 512 \times 3$ and then fed into the network for testing. To test H-DenseUNet, we inference the volume with the input size $512 \times 512 \times 8$. The final result is the concatenation of the prediction. We use the thresholding to get the kidney and tumor segmentation result, with 0.5 for kidney and 0.9 for tumor. During the post-processing, we select the largest two connected region as the kidney. We also remove the tumor prediction outside the kidney and finally we fill the holes in the final prediction. The total processing time of one subject depends on the number of slices, ranging from 30 seconds to 200 seconds. 
Table 1. Dataset split in our experiments.

\begin{tabular}{c|c|c}
\hline Dataset Split & Training Set & Validation Set \\
\hline ID & $0-199$ & $200-209$ \\
\hline
\end{tabular}

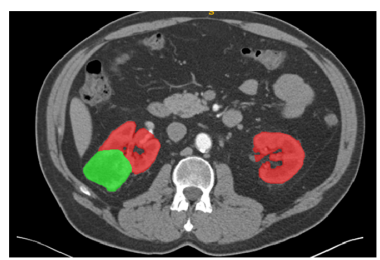

Ground truth

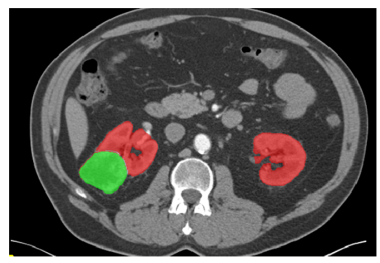

Segmentation result

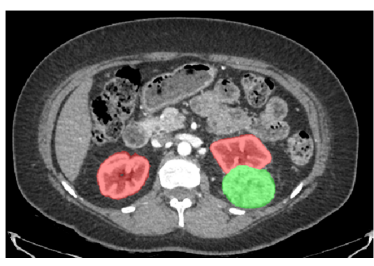

Ground truth

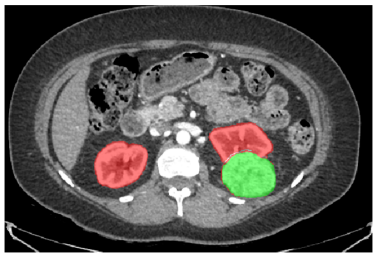

Segmentation result

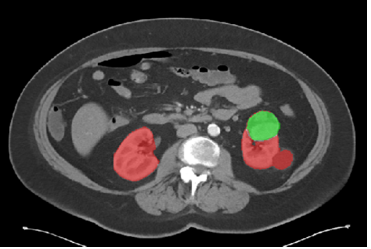

Ground truth

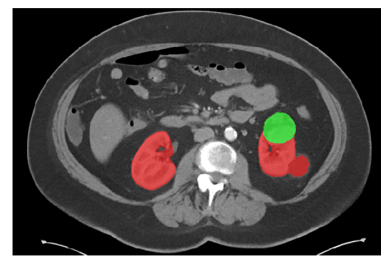

Segmentation result

Fig. 1. Examples of segmentation results of our method on the validation set. Red contour denotes the kidney and green contour denotes tumor.

\section{Results}

We employ the MICCAI 2019 kidney and tumor segmentation challenge dataset [1, $3]$. The released training data consists of $210 \mathrm{CT}$ scans with labels. In our experiments, 10 data with ID 200-209 are used for validation and the left 200 data are used for training, as shown in Table 1. The averaged Dice are employed to measure both kidney and tumor segmentation results. Our model achieves $97.0 \%$ on kidney $67.2 \%$ for tumor on the validation set. We visualize our result in Fig. 1. Our model is submitted to the MICCAI 2019 kidney and tumor segmentation for final evaluation on the test dataset.

\section{Conclusion}

In this work, we employed the H-DenseUNet [4] for the kidney and tumor segmentation. Our preliminary results on the validation set is $97.0 \%$ for kidney and $67.2 \%$ for tumor. Our model is submitted to the MICCAI 2019 kidney and tumor segmentation for final evaluation on the test dataset. 


\section{References}

1. Heller, N., Sathianathen, N., Kalapara, A., Walczak, E., Moore, K., Kaluzniak, H., Rosenberg, J., Blake, P., Rengel, Z., Oestreich, M., et al.: The kits19 challenge data: 300 kidney tumor cases with clinical context, ct semantic segmentations, and surgical outcomes. arXiv preprint arXiv:1904.00445 (2019)

2. Kingma, D.P., Ba, J.: Adam: A method for stochastic optimization. arXiv preprint arXiv:1412.6980 (2014)

3. Kutikov, A., Uzzo, R.G.: The renal nephrometry score: a comprehensive standardized system for quantitating renal tumor size, location and depth. The Journal of urology 182(3), 844-853 (2009)

4. Li, X., Chen, H., Qi, X., Dou, Q., Fu, C.W., Heng, P.A.: H-denseunet: hybrid densely connected unet for liver and tumor segmentation from ct volumes. IEEE transactions on medical imaging 37(12), 2663-2674 (2018)

5. Siegel, R.L., Miller, K.D., Jemal, A.: Cancer statistics, 2019. CA: a cancer journal for clinicians 69(1), 7-34 (2019) 